\title{
Eigenvalues for iterative systems of nonlinear $m$-point boundary value problems on time scales
}

Ilkay Y Karaca ${ }^{1 *}$ and Fatma Tokmak ${ }^{1,2}$

\section{"Correspondence:}

ilkay.karaca@ege.edu.tr

${ }^{1}$ Department of Mathematics, Ege

University, Bornova, Izmir, 35100,

Turkey

Full list of author information is

available at the end of the article

\begin{abstract}
In this paper, we determine the eigenvalue intervals of the parameters $\lambda_{1}, \lambda_{2}, \ldots, \lambda_{n}$ for which there exist positive solutions of the iterative systems of $m$-point boundary value problems on time scales. The method involves an application of Guo-Krasnosel'skii fixed point theorem. We give an example to demonstrate our main results.
\end{abstract}

MSC: 34B18; 34N05

Keywords: Green's function; iterative system; eigenvalue interval; time scales; boundary value problem; fixed point theorem; m-point; positive solution

\section{Introduction}

The study of dynamic equations on time scales goes back to Stefan Hilger [1]. Theoretically, this new theory has not only unify continuous and discrete equations, but it has also exhibited much more complicated dynamics on time scales. Moreover, the study of dynamic equations on time scales has led to several important applications, for example, insect population models, biology, neural networks, heat transfer, and epidemic models; see [2-7].

There has been much interest shown in obtaining optimal eigenvalue intervals for the existence of positive solutions of the boundary value problems on time scales, often using Guo-Krasnosel'skii fixed point theorem. To mention a few papers along these lines, see [812 ]. On the other hand, there is not much work concerning the eigenvalues for iterative system of nonlinear boundary value problems on time scales; see [13, 14].

In [15], Ma and Thompson are concerned with determining values $\lambda$, by using the GuoKrasnosel'skii fixed point theorem for which there exist positive solutions of the $m$-point boundary value problem

$$
\left\{\begin{array}{l}
\left(p(t) u^{\prime}\right)^{\prime}-q(t) u+\lambda f(t, u)=0, \quad 0<t<1, \\
a u(0)-b p(0) u^{\prime}(0)=\sum_{i=1}^{m-2} \alpha_{i} u\left(\xi_{i}\right), \\
c u(1)+d p(1) u^{\prime}(1)=\sum_{i=1}^{m-2} \beta_{i} u\left(\xi_{i}\right) .
\end{array}\right.
$$

In [13], Benchohra et al. studied the eigenvalues for iterative system of nonlinear boundary value problems on time scales,

$$
u_{i}^{\triangle \triangle}(t)+\lambda_{i} a_{i}(t) f_{i}\left(u_{i+1}(\sigma(t))\right)=0, \quad 1 \leq i \leq n, t \in[0,1]_{\mathbb{T}}
$$

○2014 Karaca and Tokmak; licensee Springer. This is an Open Access article distributed under the terms of the Creative Commons Attribution License (http://creativecommons.org/licenses/by/2.0), which permits unrestricted use, distribution, and reproduction in any medium, provided the original work is properly cited. 


$$
u_{n+1}(t)=u_{1}(t), \quad t \in[0,1]_{\mathbb{T}},
$$

satisfying the boundary conditions,

$$
u_{i}(0)=0=u_{i}\left(\sigma^{2}(1)\right), \quad 1 \leq i \leq n
$$

The method involves application of Guo-Krasnosel'skii fixed point theorem for operators on a cone in a Banach space.

In [14], Prasad et al. studied the eigenvalues for iterative system of nonlinear boundary value problems on time scales,

$$
\begin{aligned}
& y_{i}^{\Delta \Delta}(t)+\lambda_{i} p_{i}(t) f_{i}\left(y_{i+1}(t)\right)=0, \quad 1 \leq i \leq n, t \in\left[t_{1}, t_{m}\right]_{\mathbb{T}}, \\
& y_{n+1}(t)=y_{1}(t), \quad t \in\left[t_{1}, t_{m}\right]_{\mathbb{T}},
\end{aligned}
$$

satisfying the $m$-point boundary conditions,

$$
\begin{aligned}
& y_{i}\left(t_{1}\right)=0, \\
& \alpha y_{i}\left(\sigma\left(t_{m}\right)\right)+\beta y_{i}^{\triangle}\left(\sigma\left(t_{m}\right)\right)=\sum_{k=2}^{m-1} y_{i}^{\triangle}\left(t_{k}\right), \quad 1 \leq i \leq n .
\end{aligned}
$$

They used the Guo-Krasnosel'skii fixed point theorem.

Motivated by the above results, in this study, we are concerned with determining the eigenvalue intervals of $\lambda_{i}, 1 \leq i \leq n$, for which there exist positive solutions for the iterative system of nonlinear $m$-point boundary value problems on time scales,

$$
\left\{\begin{array}{l}
u_{i}^{\Delta \Delta}(t)+\lambda_{i} q_{i}(t) f_{i}\left(u_{i+1}(t)\right)=0, \quad t \in[0,1]_{\mathbb{T}}, 1 \leq i \leq n \\
u_{n+1}(t)=u_{1}(t), \quad t \in[0,1]_{\mathbb{T}}
\end{array}\right.
$$

satisfying the $m$-point boundary conditions,

$$
\left\{\begin{array}{l}
a u_{i}(0)-b u_{i}^{\Delta}(0)=\sum_{j=1}^{m-2} \alpha_{j} u_{i}\left(\xi_{j}\right), \\
c u_{i}(1)+d u_{i}^{\triangle}(1)=\sum_{j=1}^{m-2} \beta_{j} u_{i}\left(\xi_{j}\right), \quad 1 \leq i \leq n,
\end{array}\right.
$$

where $\mathbb{T}$ is a time scale, $0,1 \in \mathbb{T},[0,1]_{\mathbb{T}}=[0,1] \cap \mathbb{T}$.

Throughout this paper we assume that following conditions hold:

(C1) $a, b, c, d \in[0, \infty)$ with $a c+a d+b c>0 ; \alpha_{j}, \beta_{j} \in[0, \infty), \xi_{j} \in(0,1)_{\mathbb{T}}$ for $1 \leq j \leq m-2$,

(C2) $f_{i}: \mathbb{R}^{+} \rightarrow \mathbb{R}^{+}$is continuous, for $1 \leq i \leq n$,

(C3) $q_{i} \in \mathcal{C}\left([0,1]_{\mathbb{T}}, \mathbb{R}^{+}\right)$and $q_{i}$ does not vanish identically on any closed subinterval of $[0,1]_{\mathbb{T}}$, for $1 \leq i \leq n$,

(C4) each of $f_{i 0}:=\lim _{x \rightarrow 0^{+}} \frac{f_{i}(x)}{x}$ and $f_{i \infty}:=\lim _{x \rightarrow \infty} \frac{f_{i}(x)}{x}, 1 \leq i \leq n$, exists as positive real number.

In fact, our results are also new when $\mathbb{T}=\mathbb{R}$ (the differential case) and $\mathbb{T}=\mathbb{Z}$ (the discrete case). Therefore, the results can be considered as a contribution to this field.

This paper is organized as follows. In Section 2, we construct the Green's function for the homogeneous problem corresponding to (1.1)-(1.2) and estimate bounds for the Green's 
function. In Section 3, we determine the eigenvalue intervals for which there exist positive solutions of the boundary value problem (1.1)-(1.2) by using the Guo-Krasnosel'skii fixed point theorem for operators on a cone in a Banach space. Finally, in Section 4, we give an example to demonstrate our main results.

\section{Preliminaries}

We need the auxiliary lemmas that will be used to prove our main results.

We define $\mathbb{B}=\mathcal{C}[0,1]$, which is a Banach space with the norm

$$
\|u\|=\sup _{t \in[0,1]_{\mathbb{T}}}|u(t)|
$$

Let $h \in \mathcal{C}[0,1]$, then we consider the following boundary value problem:

$$
\left\{\begin{array}{l}
-u_{1}^{\Delta \Delta}(t)=h(t), \quad t \in[0,1]_{\mathbb{T}} \\
a u_{1}(0)-b u_{1}^{\Delta}(0)=\sum_{j=1}^{m-2} \alpha_{j} u_{1}\left(\xi_{j}\right) \\
c u_{1}(1)+d u_{1}^{\Delta}(1)=\sum_{j=1}^{m-2} \beta_{j} u_{1}\left(\xi_{j}\right)
\end{array}\right.
$$

Denote by $\theta$ and $\varphi$, the solutions of the corresponding homogeneous equation

$$
-u_{1}^{\Delta \triangle}(t)=0, \quad t \in[0,1]_{\mathbb{T}}
$$

under the initial conditions

$$
\begin{cases}\theta(0)=b, & \theta^{\Delta}(0)=a, \\ \varphi(1)=d, & \varphi^{\Delta}(1)=-c .\end{cases}
$$

Using the initial conditions (2.3), we can deduce from equation (2.2) for $\theta$ and $\varphi$ the following equations:

$$
\theta(t)=b+a t, \quad \varphi(t)=d+c(1-t) .
$$

Set

$$
\Delta:=\left|\begin{array}{cc}
-\sum_{j=1}^{m-2} \alpha_{j}\left(b+a \xi_{j}\right) & \rho-\sum_{j=1}^{m-2} \alpha_{j}\left(d+c\left(1-\xi_{j}\right)\right) \\
\rho-\sum_{j=1}^{m-2} \beta_{j}\left(b+a \xi_{j}\right) & -\sum_{j=1}^{m-2} \beta_{j}\left(d+c\left(1-\xi_{j}\right)\right)
\end{array}\right|
$$

and

$$
\rho:=a d+a c+b c .
$$

Lemma 2.1 Let (C1) hold. Assume that

(C5) $\Delta \neq 0$.

If $u_{1} \in \mathcal{C}[0,1]$ is a solution of the equation

$$
u_{1}(t)=\int_{0}^{1} G(t, s) h(s) \triangle s+A(h)(b+a t)+B(h)(d+c(1-t)),
$$


where

$$
\begin{aligned}
& G(t, s)=\frac{1}{\rho} \begin{cases}(b+a \sigma(s))(d+c(1-t)), & \sigma(s) \leq t, \\
(b+a t)(d+c(1-\sigma(s))), & t \leq s,\end{cases} \\
& A(h):=\frac{1}{\Delta}\left|\begin{array}{ll}
\sum_{j=1}^{m-2} \alpha_{j} \int_{0}^{1} G\left(\xi_{j}, s\right) h(s) \Delta s & \rho-\sum_{j=1}^{m-2} \alpha_{j}\left(d+c\left(1-\xi_{j}\right)\right) \\
\sum_{j=1}^{m-2} \beta_{j} \int_{0}^{1} G\left(\xi_{j}, s\right) h(s) \Delta s & -\sum_{j=1}^{m-2} \beta_{j}\left(d+c\left(1-\xi_{j}\right)\right)
\end{array}\right|
\end{aligned}
$$

and

$$
B(h):=\frac{1}{\Delta}\left|\begin{array}{cc}
-\sum_{j=1}^{m-2} \alpha_{j}\left(b+a \xi_{j}\right) & \sum_{j=1}^{m-2} \alpha_{j} \int_{0}^{1} G\left(\xi_{j}, s\right) h(s) \Delta s \\
\rho-\sum_{j=1}^{m-2} \beta_{j}\left(b+a \xi_{j}\right) & \sum_{j=1}^{m-2} \beta_{j} \int_{0}^{1} G\left(\xi_{j}, s\right) h(s) \Delta s
\end{array}\right|,
$$

then $u_{1}$ is a solution of the boundary value problem (2.1).

Proof Let $u_{1}$ satisfy the integral equation (2.7), then we have

$$
u_{1}(t)=\int_{0}^{1} G(t, s) h(s) \triangle s+A(h)(b+a t)+B(h)(d+c(1-t)),
$$

i.e.,

$$
\begin{aligned}
u_{1}(t)= & \int_{0}^{t} \frac{1}{\rho}(b+a(\sigma(s)))(d+c(1-t)) h(s) \triangle s \\
& +\int_{t}^{1} \frac{1}{\rho}(b+a t)(d+c(1-\sigma(s))) h(s) \triangle s \\
& +A(h)(b+a t)+B(h)(d+c(1-t)), \\
u_{1}^{\Delta}(t)= & -\int_{0}^{t} \frac{c}{\rho}(b+a(\sigma(s))) h(s) \triangle s \\
& +\int_{t}^{1} \frac{a}{\rho}(d+c(1-\sigma(s))) h(s) \triangle s \\
& +A(h) a-B(h) c .
\end{aligned}
$$

Hence

$$
\begin{aligned}
u_{1}^{\Delta \Delta}(t) & =\frac{1}{\rho}(-c(b+a(\sigma(t)))-a(d+c(1-\sigma(t)))) h(t) \\
& =\frac{1}{\rho}(-(a d+a c+b c)) h(t)=-h(t), \\
-u_{1}^{\Delta \Delta}(t) & =h(t) .
\end{aligned}
$$

Since

$$
\begin{aligned}
& u_{1}(0)=\int_{0}^{1} \frac{b}{\rho}(d+c(1-\sigma(s))) h(s) \triangle s+A(h) b+B(h)(d+c), \\
& u_{1}^{\Delta}(0)=\int_{0}^{1} \frac{a}{\rho}(d+c(1-\sigma(s))) h(s) \triangle s+A(h) a-B(h) c,
\end{aligned}
$$


we have

$$
\begin{aligned}
a u_{1}(0)-b u_{1}^{\Delta}(0)= & B(h) \rho \\
= & \sum_{j=1}^{m-2} \alpha_{j}\left[\int_{0}^{1} G\left(\xi_{j}, s\right) h(s) \triangle s+A(h)\left(b+a \xi_{j}\right)\right. \\
& \left.+B(h)\left(d+c\left(1-\xi_{j}\right)\right)\right] .
\end{aligned}
$$

Since

$$
\begin{aligned}
& u_{1}(1)=\int_{0}^{1} \frac{d}{\rho}(b+a(\sigma(s))) h(s) \triangle s+A(h)(b+a)+B(h) d, \\
& u_{1}^{\Delta}(1)=-\int_{0}^{1} \frac{c}{\rho}(b+a(\sigma(s))) h(s) \triangle s+A(h) a-B(h) c,
\end{aligned}
$$

we have

$$
\begin{aligned}
c u_{1}(1)+d u_{1}^{\Delta}(1)= & A(h) \rho \\
= & \sum_{j=1}^{m-2} \beta_{j}\left[\int_{0}^{1} G\left(\xi_{j}, s\right) h(s) \triangle s+A(h)\left(b+a \xi_{j}\right)\right. \\
& \left.+B(h)\left(d+c\left(1-\xi_{j}\right)\right)\right] .
\end{aligned}
$$

From (2.11) and (2.12), we get

$$
\left\{\begin{array}{l}
{\left[-\sum_{j=1}^{m-2} \alpha_{j}\left(b+a \xi_{j}\right)\right] A(h)+\left[\rho-\sum_{i=1}^{m-2} \alpha_{j}\left(d+c\left(1-\xi_{j}\right)\right)\right] B(h)} \\
\quad=\sum_{i=1}^{m-2} \alpha_{j} \int_{0}^{1} G\left(\xi_{j}, s\right) h(s) \triangle s, \\
{\left[\rho-\sum_{j=1}^{m-2} \beta_{j}\left(b+a \xi_{j}\right)\right] A(h)+\left[-\sum_{i=1}^{m-2} \beta_{j}\left(d+c\left(1-\xi_{j}\right)\right)\right] B(h)} \\
\quad=\sum_{j=1}^{m-2} \beta_{j} \int_{0}^{1} G\left(\xi_{j}, s\right) h(s) \triangle s,
\end{array}\right.
$$

which implies that $A(h)$ and $B(h)$ satisfy (2.9) and (2.10), respectively.

Lemma 2.2 Let (C1) hold. Assume

(C6) $\Delta<0, \rho-\sum_{j=1}^{m-2} \beta_{j}\left(b+a \xi_{j}\right)>0, a-\sum_{j=1}^{m-2} \alpha_{j}>0$.

Then for $u_{1} \in \mathcal{C}[0,1]$ with $h \geq 0$, the solution $u_{1}$ of the problem (2.1) satisfies

$$
u_{1}(t) \geq 0 \quad \text { for } t \in[0,1]_{\mathbb{T}} .
$$

Proof It is an immediate subsequence of the facts that $G \geq 0$ on $[0,1]_{\mathbb{T}} \times[0,1]_{\mathbb{T}}$ and $A(h) \geq$ $0, B(h) \geq 0$.

Lemma 2.3 Let (C1) and (C6) hold. Assume

(C7) $c-\sum_{j=1}^{m-2} \beta_{j}<0$.

Then the solution $u_{1} \in \mathcal{C}[0,1]$ of the problem (2.1) satisfies $u_{1}^{\Delta}(t) \geq 0$ for $t \in[0,1]_{\mathbb{T}}$. 
Proof Assume that the inequality $u_{1}^{\Delta}(t)<0$ holds. Since $u_{1}^{\Delta}(t)$ is nonincreasing on $[0,1]_{\mathbb{T}}$, one can verify that

$$
u_{1}^{\Delta}(1) \leq u_{1}^{\Delta}(t), \quad t \in[0,1]_{\mathbb{T}} .
$$

From the boundary conditions of the problem (2.1), we have

$$
-\frac{c}{d} u_{1}(1)+\frac{1}{d} \sum_{i=1}^{m-2} \beta_{i} u_{1}\left(\xi_{i}\right) \leq u_{1}^{\Delta}(t)<0 .
$$

The last inequality yields

$$
-c u_{1}(1)+\sum_{i=1}^{m-2} \beta_{i} u_{1}\left(\xi_{i}\right)<0
$$

Therefore, we obtain

$$
\sum_{i=1}^{m-2} \beta_{i} u_{1}(1)<\sum_{i=1}^{m-2} \beta_{i} u_{1}\left(\xi_{i}\right)<c u_{1}(1)
$$

i.e.,

$$
\left(c-\sum_{i=1}^{m-2} \beta_{i}\right) u_{1}(1)>0 .
$$

According to Lemma 2.2, we have $u_{1}(1) \geq 0$. So, $c-\sum_{i=1}^{m-2} \beta_{i}>0$. However, this contradicts to condition (C7). Consequently, $u_{1}^{\Delta}(t) \geq 0$ for $t \in[0,1]_{\mathbb{T}}$.

Lemma 2.4 Let $(\mathrm{C} 1)$ and $h \geq 0$ hold. Let $\mu \in(0,1 / 2)_{\mathbb{T}}$ be a constant. Then the unique solution $u_{1}$ of the problem (2.1) satisfies

$$
\min _{t \in[\mu, 1-\mu]_{\mathbb{T}}} u_{1}(t) \geq \gamma\left\|u_{1}\right\|
$$

where $\left\|u_{1}\right\|=\sup _{t \in[0,1]_{\mathbb{T}}} u_{1}(t)$ and

$$
\gamma:=\min \left\{\frac{b+a \mu}{b+a}, \frac{d+c \mu}{d+c}\right\} .
$$

Proof We have from (2.8) that

$$
0 \leq G(t, s) \leq G(\sigma(s), s), \quad t \in[0,1]_{\mathbb{T}}
$$

which implies

$$
u_{1}(t) \leq \int_{0}^{1} G(\sigma(s), s) h(s) \triangle s+A(h)(b+a)+B(h)(d+c)
$$


for all $t \in[0,1]_{\mathbb{T}}$. Applying (2.8), we have for $t \in[\mu, 1-\mu]_{\mathbb{T}}$,

$$
\begin{aligned}
\frac{G(t, s)}{G(\sigma(s), s)} & = \begin{cases}\frac{d+c(1-t)}{d+c(1-\sigma(s))}, & 0 \leq \sigma(s) \leq t \leq 1, \\
\frac{b+a t}{b+a \sigma(s)}, & 0 \leq t \leq s \leq 1\end{cases} \\
& \geq\left\{\begin{array}{ll}
\frac{d+c \mu}{d+c}, & 0 \leq \sigma(s) \leq t \leq 1-\mu, \\
\frac{b+a \mu}{b+a}, & \mu \leq t \leq s \leq 1
\end{array} \geq \gamma,\right.
\end{aligned}
$$

where

$$
\gamma:=\min \left\{\frac{b+a \mu}{b+a}, \frac{d+c \mu}{d+c}\right\} .
$$

Thus for $t \in[\mu, 1-\mu]_{\mathbb{T}}$,

$$
\begin{aligned}
u_{1}(t) & =\int_{0}^{1} G(t, s) h(s) \triangle s+A(h)(b+a t)+B(h)(d+c(1-t)) \\
& \geq \gamma\left(\int_{0}^{1} G(\sigma(s), s) h(s) \triangle s+A(h)(b+a)+B(h)(d+c)\right) \geq \gamma\left\|u_{1}\right\| .
\end{aligned}
$$

So, the proof is completed.

We note that an $n$-tuple $\left(u_{1}(t), u_{2}(t), \ldots, u_{n}(t)\right)$ is a solution of the boundary value problem (1.1)-(1.2) if and only if

$$
\begin{aligned}
u_{1}(t)= & \lambda_{1} \int_{0}^{1} G\left(t, s_{1}\right) q_{1}\left(s_{1}\right) f_{1}\left(\lambda_{2} \int_{0}^{1} G\left(s_{1}, s_{2}\right) q_{2}\left(s_{2}\right) \cdots\right. \\
& \left.f_{n-1}\left(\lambda_{n} \int_{0}^{1} G\left(s_{n-1}, s_{n}\right) q_{n}\left(s_{n}\right) f_{n}\left(u_{1}\left(s_{n}\right)\right) \triangle s_{n}\right) \cdots \triangle s_{2}\right) \triangle s_{1} \\
& +A\left(\lambda_{1} q_{1}(\cdot) f_{1}\left(u_{2}(\cdot)\right)\right)(b+a t)+B\left(\lambda_{1} q_{1}(\cdot) f_{1}\left(u_{2}(\cdot)\right)\right)(d+c(1-t)), \quad t \in[0,1]_{\mathbb{T}}, \\
u_{i}(t)= & \lambda_{i} \int_{0}^{1} G(t, s) q_{i}(s) f_{i}\left(u_{i+1}(s)\right) \triangle s+A\left(\lambda_{i} q_{i}(\cdot) f_{i}\left(u_{i+1}(\cdot)\right)\right)(b+a t) \\
& +B\left(\lambda_{i} q_{i}(\cdot) f_{i}\left(u_{i+1}(\cdot)\right)\right)(d+c(1-t)), \quad 2 \leq i \leq n, t \in[0,1]_{\mathbb{T}}
\end{aligned}
$$

and

$$
u_{n+1}(t)=u_{1}(t), \quad t \in[0,1]_{\mathbb{T}},
$$

where

$$
\begin{aligned}
& A\left(\lambda_{i} q_{i}(\cdot) f_{i}\left(u_{i+1}(\cdot)\right)\right) \\
& \quad:=\frac{1}{\Delta}\left|\begin{array}{cc}
\sum_{j=1}^{m-2} \alpha_{j} \lambda_{i} \int_{0}^{1} G\left(\xi_{j}, s\right) q_{i}(s) f_{i}\left(u_{i+1}(s)\right) \triangle s & \rho-\sum_{j=1}^{m-2} \alpha_{j}\left(d+c\left(1-\xi_{j}\right)\right) \\
\sum_{j=1}^{m-2} \beta_{j} \lambda_{i} \int_{0}^{1} G\left(\xi_{j}, s\right) q_{i}(s) f_{i}\left(u_{i+1}(s)\right) \Delta s & -\sum_{j=1}^{m-2} \beta_{j}\left(d+c\left(1-\xi_{j}\right)\right)
\end{array}\right|, \\
& B\left(\lambda_{i} q_{i}(\cdot) f_{i}\left(u_{i+1}(\cdot)\right)\right) \\
& \quad:=\frac{1}{\Delta}\left|\begin{array}{cc}
-\sum_{j=1}^{m-2} \alpha_{j}\left(b+a \xi_{j}\right) & \sum_{j=1}^{m-2} \alpha_{j} \lambda_{i} \int_{0}^{1} G\left(\xi_{j}, s\right) q_{i}(s) f_{i}\left(u_{i+1}(s)\right) \Delta s \\
\rho-\sum_{j=1}^{m-2} \beta_{j}\left(b+a \xi_{j}\right) & \sum_{j=1}^{m-2} \beta_{j} \lambda_{i} \int_{0}^{1} G\left(\xi_{j}, s\right) q_{i}(s) f_{i}\left(u_{i+1}(s)\right) \Delta s
\end{array}\right| .
\end{aligned}
$$


To determine the eigenvalue intervals of the boundary value problem (1.1)-(1.2), we will use the following Guo-Krasnosel'skii fixed point theorem [16].

Theorem 2.1 [16] Let $\mathbb{B}$ be a Banach space, and let $\mathcal{P} \subset \mathbb{B}$ be a cone in $\mathbb{B}$. Assume $\Omega_{1}$ and $\Omega_{2}$ are open subsets of $\mathbb{B}$ with $0 \in \Omega_{1}$ and $\bar{\Omega}_{1} \subset \Omega_{2}$, and let

$$
T: \mathcal{P} \cap\left(\bar{\Omega}_{2} \backslash \Omega_{1}\right) \rightarrow \mathcal{P}
$$

be a completely continuous operator such that either

(i) $\|T u\| \leq\|u\|, u \in \mathcal{P} \cap \partial \Omega_{1}$, and $\|T u\| \geq\|u\|, u \in \mathcal{P} \cap \partial \Omega_{2}$, or

(ii) $\|T u\| \geq\|u\|, u \in \mathcal{P} \cap \partial \Omega_{1}$, and $\|T u\| \leq\|u\|, u \in \mathcal{P} \cap \partial \Omega_{2}$.

Then $T$ has a fixed point in $\mathcal{P} \cap\left(\bar{\Omega}_{2} \backslash \Omega_{1}\right)$.

\section{Positive solutions in a cone}

In this section, we establish criteria to determine the eigenvalue intervals for which the boundary value problem (1.1)-(1.2) has at least one positive solution in a cone. We construct a cone $\mathcal{P} \subset \mathbb{B}$ by

$$
\mathcal{P}=\left\{u \in \mathbb{B}: u(t) \geq 0 \text { on }[0,1]_{\mathbb{T}} \text { and } \min _{t \in[\mu, 1-\mu]_{\mathbb{T}}} u(t) \geq \gamma\|u\|\right\},
$$

where $\gamma$ is given in (2.13).

Now, we define an integral operator $T: \mathcal{P} \rightarrow \mathbb{B}$, for $u_{1} \in \mathcal{P}$, by

$$
\begin{aligned}
T u_{1}(t)= & \lambda_{1} \int_{0}^{1} G\left(t, s_{1}\right) q_{1}\left(s_{1}\right) f_{1}\left(\lambda_{2} \int_{0}^{1} G\left(s_{1}, s_{2}\right) q_{2}\left(s_{2}\right) \cdots\right. \\
& \left.f_{n-1}\left(\lambda_{n} \int_{0}^{1} G\left(s_{n-1}, s_{n}\right) q_{n}\left(s_{n}\right) f_{n}\left(u_{1}\left(s_{n}\right)\right) \triangle s_{n}\right) \cdots \triangle s_{2}\right) \triangle s_{1} \\
& +A\left(\lambda_{1} q_{1}(\cdot) f_{1}\left(u_{2}(\cdot)\right)\right)(b+a t)+B\left(\lambda_{1} q_{1}(\cdot) f_{1}\left(u_{2}(\cdot)\right)\right)(d+c(1-t)) .
\end{aligned}
$$

Notice from (C1)-(C6) and Lemma 2.2 that, for $u_{1} \in \mathcal{P}, T u_{1}(t) \geq 0$ on $t \in[0,1]_{\mathbb{T}}$. Also, we have from (2.8), that

$$
\begin{aligned}
T u_{1}(t) \leq & \lambda_{1} \int_{0}^{1} G\left(\sigma\left(s_{1}\right), s_{1}\right) q_{1}\left(s_{1}\right) f_{1}\left(\lambda_{2} \int_{0}^{1} G\left(s_{1}, s_{2}\right) q_{2}\left(s_{2}\right) \cdots\right. \\
& \left.f_{n-1}\left(\lambda_{n} \int_{0}^{1} G\left(s_{n-1}, s_{n}\right) q_{n}\left(s_{n}\right) f_{n}\left(u_{1}\left(s_{n}\right)\right) \triangle s_{n}\right) \cdots \Delta s_{2}\right) \triangle s_{1} \\
& +A\left(\lambda_{1} q_{1}(\cdot) f_{1}\left(u_{2}(\cdot)\right)\right)(b+a)+B\left(\lambda_{1} q_{1}(\cdot) f_{1}\left(u_{2}(\cdot)\right)\right)(d+c),
\end{aligned}
$$

so that

$$
\begin{aligned}
\left\|T u_{1}\right\| \leq & \lambda_{1} \int_{0}^{1} G\left(\sigma\left(s_{1}\right), s_{1}\right) q_{1}\left(s_{1}\right) f_{1}\left(\lambda_{2} \int_{0}^{1} G\left(s_{1}, s_{2}\right) q_{2}\left(s_{2}\right) \cdots\right. \\
& \left.f_{n-1}\left(\lambda_{n} \int_{0}^{1} G\left(s_{n-1}, s_{n}\right) q_{n}\left(s_{n}\right) f_{n}\left(u_{1}\left(s_{n}\right)\right) \triangle s_{n}\right) \cdots \Delta s_{2}\right) \triangle s_{1} \\
& +A\left(\lambda_{1} q_{1}(\cdot) f_{1}\left(u_{2}(\cdot)\right)\right)(b+a)+B\left(\lambda_{1} q_{1}(\cdot) f_{1}\left(u_{2}(\cdot)\right)\right)(d+c) .
\end{aligned}
$$


Next, if $u_{1} \in \mathcal{P}$, we have from Lemma 2.4 and (3.2) that

$$
\begin{array}{rl}
\min _{t \in[\mu, 1-\mu]_{\mathbb{T}}} & T u_{1}(t) \\
= & \min _{t \in[\mu, 1-\mu]_{\mathbb{T}}}\left\{\lambda _ { 1 } \int _ { 0 } ^ { 1 } G ( t , s _ { 1 } ) q _ { 1 } ( s _ { 1 } ) f _ { 1 } \left(\lambda_{2} \int_{0}^{1} G\left(s_{1}, s_{2}\right) q_{2}\left(s_{2}\right) \cdots\right.\right. \\
& \left.f_{n-1}\left(\lambda_{n} \int_{0}^{1} G\left(s_{n-1}, s_{n}\right) q_{n}\left(s_{n}\right) f_{n}\left(u_{1}\left(s_{n}\right)\right) \triangle s_{n}\right) \cdots \Delta s_{2}\right) \triangle s_{1} \\
& \left.+A\left(\lambda_{1} q_{1}(\cdot) f_{1}\left(u_{2}(\cdot)\right)\right)(b+a t)+B\left(\lambda_{1} q_{1}(\cdot) f_{1}\left(u_{2}(\cdot)\right)\right)(d+c(1-t))\right\} \\
\geq & \gamma\left(\lambda _ { 1 } \int _ { 0 } ^ { 1 } G ( \sigma ( s _ { 1 } ) , s _ { 1 } ) q _ { 1 } ( s _ { 1 } ) f _ { 1 } \left(\lambda_{2} \int_{0}^{1} G\left(s_{1}, s_{2}\right) q_{2}\left(s_{2}\right) \cdots\right.\right. \\
& \left.f_{n-1}\left(\lambda_{n} \int_{0}^{1} G\left(s_{n-1}, s_{n}\right) q_{n}\left(s_{n}\right) f_{n}\left(u_{1}\left(s_{n}\right)\right) \triangle s_{n}\right) \cdots \Delta s_{2}\right) \triangle s_{1} \\
& \left.+A\left(\lambda_{1} q_{1}(\cdot) f_{1}\left(u_{2}(\cdot)\right)\right)(b+a)+B\left(\lambda_{1} q_{1}(\cdot) f_{1}\left(u_{2}(\cdot)\right)\right)(d+c)\right) \\
\geq & \gamma\left\|T u_{1}\right\| .
\end{array}
$$

Hence, $T u_{1} \in \mathcal{P}$ and $T: \mathcal{P} \rightarrow \mathcal{P}$. In addition, the operator $T$ is completely continuous by an application of the Arzela-Ascoli theorem.

Now, we investigate suitable fixed points of $T$ belonging to the cone $\mathcal{P}$. For convenience we introduce the following notations.

Let

$$
M_{1}=\max _{1 \leq i \leq n}\left\{\left[\gamma^{2} \int_{\mu}^{1-\mu} G(\sigma(s), s) q_{i}(s) \Delta s f_{i \infty}\right]^{-1}\right\}
$$

and

$$
M_{2}=\min _{1 \leq i \leq n}\left\{\left[\left(\int_{0}^{1} G(\sigma(s), s) q_{i}(s) \triangle s+A\left(q_{i}(\cdot)\right)(b+a)+B\left(q_{i}(\cdot)\right)(d+c)\right) f_{i 0}\right]^{-1}\right\} .
$$

Theorem 3.1 Suppose conditions (C1)-(C7) are satisfied. Then, for each $\lambda_{1}, \lambda_{2}, \ldots, \lambda_{n}$ satisfying

$$
M_{1}<\lambda_{i}<M_{2}, \quad 1 \leq i \leq n,
$$

there exists an $n$-tuple $\left(u_{1}, u_{2}, \ldots, u_{n}\right)$ satisfying (1.1)-(1.2) such that $u_{i}(t)>0,1 \leq i \leq n$, on $[0,1]_{\mathbb{T}}$.

Proof Let $\lambda_{k}, 1 \leq k \leq n$, be as in (3.3). Now, let $\epsilon>0$ be chosen such that

$$
\max _{1 \leq i \leq n}\left\{\left[\gamma^{2} \int_{\mu}^{1-\mu} G(\sigma(s), s) q_{i}(s) \Delta s\left(f_{i \infty}-\epsilon\right)\right]^{-1}\right\} \leq \min _{1 \leq k \leq n} \lambda_{k}
$$

and

$$
\max _{1 \leq k \leq n} \lambda_{k} \leq \min _{1 \leq i \leq n}\left\{\left[\left(\int_{0}^{1} G(\sigma(s), s) q_{i}(s) \triangle s+A\left(q_{i}(\cdot)\right)(b+a)+B\left(q_{i}(\cdot)\right)(d+c)\right)\left(f_{i 0}+\epsilon\right)\right]^{-1}\right\} .
$$


We investigate fixed points of the completely continuous operator $T: \mathcal{P} \rightarrow \mathcal{P}$ defined by (3.1). Now, from the definitions of $f_{i 0}, 1 \leq i \leq n$, there exists an $H_{1}>0$ such that, for each $1 \leq i \leq n$,

$$
f_{i}(x) \leq\left(f_{i 0}+\epsilon\right) x, \quad 0<x \leq H_{1} .
$$

Let $u_{1} \in \mathcal{P}$ with $\left\|u_{1}\right\|=H_{1}$. We have from (2.14) and the choice of $\epsilon$, for $0 \leq s_{n-1} \leq 1$,

$$
\begin{aligned}
& \lambda_{n} \int_{0}^{1} G\left(s_{n-1}, s_{n}\right) q_{n}\left(s_{n}\right) f_{n}\left(u_{1}\left(s_{n}\right)\right) \triangle s_{n} \\
& \quad \leq \lambda_{n} \int_{0}^{1} G\left(\sigma\left(s_{n}\right), s_{n}\right) q_{n}\left(s_{n}\right) f_{n}\left(u_{1}\left(s_{n}\right)\right) \triangle s_{n} \\
& \quad \leq \lambda_{n} \int_{0}^{1} G\left(\sigma\left(s_{n}\right), s_{n}\right) q_{n}\left(s_{n}\right)\left(f_{n 0}+\epsilon\right) u_{1}\left(s_{n}\right) \triangle s_{n} \\
& \quad \leq \lambda_{n} \int_{0}^{1} G\left(\sigma\left(s_{n}\right), s_{n}\right) q_{n}\left(s_{n}\right) \triangle s_{n}\left(f_{n 0}+\epsilon\right)\left\|u_{1}\right\| \\
& \quad \leq\left\|u_{1}\right\| \\
& \quad \leq H_{1} .
\end{aligned}
$$

It follows in a similar manner from (2.14), for $0 \leq s_{n-2} \leq 1$, that

$$
\begin{aligned}
& \lambda_{n-1} \int_{0}^{1} G\left(s_{n-2}, s_{n-1}\right) q_{n-1}\left(s_{n-1}\right) f_{n-1}\left(\lambda_{n} \int_{0}^{1} G\left(s_{n-1}, s_{n}\right) q_{n}\left(s_{n}\right) f_{n}\left(u_{1}\left(s_{n}\right)\right) \triangle s_{n}\right) \triangle s_{n-1} \\
& \quad \leq \lambda_{n-1} \int_{0}^{1} G\left(\sigma\left(s_{n-1}\right), s_{n-1}\right) q_{n-1}\left(s_{n-1}\right) \triangle s_{n-1}\left(f_{n-1,0}+\epsilon\right)\left\|u_{1}\right\| \\
& \quad \leq\left\|u_{1}\right\|=H_{1} .
\end{aligned}
$$

Continuing with this bootstrapping argument, we have, for $0 \leq t \leq 1$,

$$
\begin{aligned}
& \lambda_{1} \int_{0}^{1} G\left(t, s_{1}\right) q_{1}\left(s_{1}\right) f_{1}\left(\lambda_{2} \int_{0}^{1} G\left(s_{1}, s_{2}\right) q_{2}\left(s_{2}\right) \cdots f_{n}\left(u_{1}\left(s_{n}\right)\right) \triangle s_{n} \cdots \Delta s_{2}\right) \triangle s_{1} \\
& \quad \leq \lambda_{1} \int_{0}^{1} G\left(\sigma\left(s_{1}\right), s_{1}\right) q_{1}\left(s_{1}\right) \triangle s_{1}\left(f_{10}+\epsilon\right) H_{1}, \\
& A\left(\lambda_{1} q_{1}(\cdot) f_{1}\left(u_{2}(\cdot)\right)\right) \\
& \quad \leq \frac{\lambda_{1}}{\Delta}\left|\begin{array}{ll}
\sum_{j=1}^{m-2} \alpha_{j} \int_{0}^{1} G\left(\xi_{j}, s\right) q_{1}(s) \triangle s & \rho-\sum_{j=1}^{m-2} \alpha_{j}\left(d+c\left(1-\xi_{j}\right)\right) \\
\sum_{j=1}^{m-2} \beta_{j} \int_{0}^{1} G\left(\xi_{j}, s\right) q_{1}(s) \Delta s & -\sum_{j=1}^{m-2} \beta_{j}\left(d+c\left(1-\xi_{j}\right)\right)
\end{array}\right|\left\|f_{1}\left(u_{2}\right)\right\| \\
& \quad \leq \lambda_{1} A\left(q_{1}(\cdot)\right)\left\|f_{1}\left(u_{2}\right)\right\|, \\
& B\left(\lambda_{1} q_{1}(\cdot) f_{1}\left(u_{2}(\cdot)\right)\right) \\
& \quad \leq \frac{\lambda_{1}}{\Delta}\left|\begin{array}{ll}
-\sum_{j=1}^{m-2} \alpha_{j}\left(b+a\left(\xi_{j}\right)\right) & \sum_{j=1}^{m-2} \alpha_{j} \int_{0}^{1} G\left(\xi_{j}, s\right) q_{1}(s) \Delta s \\
\rho-\sum_{j=1}^{m-2} \beta_{j}\left(b+a\left(\xi_{j}\right)\right) & \sum_{j=1}^{m-2} \beta_{j} \int_{0}^{1} G\left(\xi_{j}, s\right) q_{1}(s) \Delta s
\end{array}\right|\left\|f_{1}\left(u_{2}\right)\right\| \\
& \quad \leq \lambda_{1} B\left(q_{1}(\cdot)\right)\left\|f_{1}\left(u_{2}\right)\right\|,
\end{aligned}
$$


so that, for $0 \leq t \leq 1$,

$$
\begin{aligned}
T u_{1}(t) \leq & \lambda_{1}\left(\int_{0}^{1} G\left(\sigma\left(s_{1}\right), s_{1}\right) q_{1}\left(s_{1}\right) \triangle s_{1}\left(f_{10}+\epsilon\right) H_{1}\right. \\
& \left.+A\left(q_{1}(\cdot)\right)\left\|f_{1}\left(u_{2}\right)\right\|(b+a)+B\left(q_{1}(\cdot)\right)\left\|f_{1}\left(u_{2}\right)\right\|(d+c)\right) \\
\leq & \lambda_{1}\left(\int_{0}^{1} G\left(\sigma\left(s_{1}\right), s_{1}\right) q_{1}\left(s_{1}\right) \triangle s_{1}\right. \\
& \left.+A\left(q_{1}(\cdot)\right)(b+a)+B\left(q_{1}(\cdot)\right)(d+c)\right)\left(f_{10}+\epsilon\right) H_{1} \\
\leq & H_{1}=\left\|u_{1}\right\| .
\end{aligned}
$$

Hence, $\left\|T u_{1}\right\| \leq H_{1}=\left\|u_{1}\right\|$. If we set

$$
\Omega_{1}=\left\{u \in \mathbb{B} \mid\|u\|<H_{1}\right\},
$$

then

$$
\left\|T u_{1}\right\| \leq\left\|u_{1}\right\| \quad \text { for } u_{1} \in \mathcal{P} \cap \partial \Omega_{1} .
$$

Next, from the definitions of $f_{i \infty}, 1 \leq i \leq n$, there exists $\bar{H}_{2}>0$ such that, for each $1 \leq$ $i \leq n$,

$$
f_{i}(x) \geq\left(f_{i \infty}-\epsilon\right) x, \quad x \geq \bar{H}_{2}
$$

Let

$$
H_{2}=\max \left\{2 H_{1}, \frac{\bar{H}_{2}}{\gamma}\right\}
$$

Let $u_{1} \in \mathcal{P}$ and $\left\|u_{1}\right\|=H_{2}$. Then, we have from Lemma 2.4

$$
\min _{t \in[\mu, 1-\mu]_{\mathbb{T}}} u_{1}(t) \geq \gamma\left\|u_{1}\right\| \geq \bar{H}_{2}
$$

Consequently, from Lemma 2.4 and the choice of $\epsilon$, for $0 \leq s_{n-1} \leq 1$, we have

$$
\begin{aligned}
& \lambda_{n} \int_{0}^{1} G\left(s_{n-1}, s_{n}\right) q_{n}\left(s_{n}\right) f_{n}\left(u_{1}\left(s_{n}\right)\right) \triangle s_{n} \\
& \quad \geq \gamma \lambda_{n} \int_{\mu}^{1-\mu} G\left(\sigma\left(s_{n}\right), s_{n}\right) q_{n}\left(s_{n}\right) f_{n}\left(u_{1}\left(s_{n}\right)\right) \triangle s_{n} \\
& \quad \geq \gamma \lambda_{n} \int_{\mu}^{1-\mu} G\left(\sigma\left(s_{n}\right), s_{n}\right) q_{n}\left(s_{n}\right)\left(f_{n \infty}-\epsilon\right) u_{1}\left(s_{n}\right) \triangle s_{n} \\
& \quad \geq \gamma^{2} \lambda_{n} \int_{\mu}^{1-\mu} G\left(\sigma\left(s_{n}\right), s_{n}\right) q_{n}\left(s_{n}\right) \triangle s_{n}\left(f_{n \infty}-\epsilon\right)\left\|u_{1}\right\| \\
& \quad \geq\left\|u_{1}\right\|=H_{2} .
\end{aligned}
$$


It follows in a similar manner from Lemma 2.4 and the choice of $\epsilon$, for $0 \leq s_{n-2} \leq 1$,

$$
\begin{aligned}
& \lambda_{n-1} \int_{0}^{1} G\left(s_{n-2}, s_{n-1}\right) q_{n-1}\left(s_{n-1}\right) f_{n-1}\left(\lambda_{n} \int_{0}^{1} G\left(s_{n-1}, s_{n}\right) q_{n}\left(s_{n}\right) f_{n}\left(u_{1}\left(s_{n}\right)\right) \Delta s_{n}\right) \triangle s_{n-1} \\
& \quad \geq \gamma \lambda_{n-1} \int_{\mu}^{1-\mu} G\left(\sigma\left(s_{n-1}\right), s_{n-1}\right) q_{n-1}\left(s_{n-1}\right) \triangle s_{n-1}\left(f_{n-1, \infty}-\epsilon\right) H_{2} \\
& \quad \geq \gamma^{2} \lambda_{n-1} \int_{\mu}^{1-\mu} G\left(\sigma\left(s_{n-1}\right), s_{n-1}\right) q_{n-1}\left(s_{n-1}\right) \triangle s_{n-1}\left(f_{n-1, \infty}-\epsilon\right) H_{2} \\
& \geq H_{2} .
\end{aligned}
$$

Again, using a bootstrapping argument, we have

$$
\begin{aligned}
& \lambda_{1} \int_{0}^{1} G\left(t, s_{1}\right) q_{1}\left(s_{1}\right) f_{1}\left(\lambda_{2} \int_{0}^{1} G\left(s_{1}, s_{2}\right) q_{2}\left(s_{2}\right) \cdots f_{n}\left(u_{1}\left(s_{n}\right)\right) \Delta s_{n} \cdots \Delta s_{2}\right) \triangle s_{1} \\
& \quad \geq H_{2}
\end{aligned}
$$

so that

$$
T u_{1}(t) \geq H_{2}=\left\|u_{1}\right\|
$$

Hence, $\left\|T u_{1}\right\| \geq\left\|u_{1}\right\|$. So if we set

$$
\Omega_{2}=\left\{u \in \mathbb{B} \mid\|u\|<H_{2}\right\}
$$

then

$$
\left\|T u_{1}\right\| \geq\left\|u_{1}\right\| \quad \text { for } u_{1} \in \mathcal{P} \cap \partial \Omega_{2} .
$$

Applying Theorem 2.1 to (3.4) and (3.5), we see that $T$ has a fixed point $u_{1} \in \mathcal{P} \cap\left(\bar{\Omega}_{2} \backslash \Omega_{1}\right)$. Therefore, setting $u_{n+1}=u_{1}$, we obtain a positive solution $\left(u_{1}, u_{2}, \ldots, u_{n}\right)$ of (1.1)-(1.2) given iteratively by

$$
\begin{aligned}
u_{k}(t)= & \lambda_{k} \int_{0}^{1} G(t, s) q_{k}(s) f_{k}\left(u_{k+1}(s)\right) \triangle s+A\left(\lambda_{k} q_{k}(\cdot) f_{k}\left(u_{k+1}(\cdot)\right)\right)(b+a t) \\
& +B\left(\lambda_{k} q_{k}(\cdot) f_{k}\left(u_{k+1}(\cdot)\right)\right)(d+c(1-t)), \quad k=n, n-1, \ldots, 1 .
\end{aligned}
$$

The proof is completed.

For our next result, we define the positive numbers $M_{3}$ and $M_{4}$ by

$$
M_{3}=\max _{1 \leq i \leq n}\left\{\left[\gamma^{2} \int_{\mu}^{1-\mu} G(\sigma(s), s) q_{i}(s) \triangle s f_{i 0}\right]^{-1}\right\}
$$

and

$$
M_{4}=\min _{1 \leq i \leq n}\left\{\left[\left(\int_{0}^{1} G(\sigma(s), s) q_{i}(s) \triangle s+A\left(q_{i}(\cdot)\right)(b+a)+B\left(q_{i}(\cdot)\right)(d+c)\right) f_{i \infty}\right]^{-1}\right\} .
$$


Theorem 3.2 Suppose conditions (C1)-(C7) are satisfied. Then, for each $\lambda_{1}, \lambda_{2}, \ldots, \lambda_{n}$ satisfying

$$
M_{3}<\lambda_{i}<M_{4}, \quad 1 \leq i \leq n,
$$

there exists an $n$-tuple $\left(u_{1}, u_{2}, \ldots, u_{n}\right)$ satisfying (1.1)-(1.2) such that $u_{i}(t)>0,1 \leq i \leq n$, on $[0,1]_{\mathbb{T}}$.

Proof Let $\lambda_{k}, 1 \leq k \leq n$, be as in (3.6). Now, let $\epsilon>0$ be chosen such that

$$
\max _{1 \leq i \leq n}\left\{\left[\gamma^{2} \int_{\mu}^{1-\mu} G(\sigma(s), s) q_{i}(s) \triangle s\left(f_{i 0}-\epsilon\right)\right]^{-1}\right\} \leq \min _{1 \leq k \leq n} \lambda_{k}
$$

and

$$
\max _{1 \leq k \leq n} \lambda_{k} \leq \min _{1 \leq i \leq n}\left\{\left[\left(\int_{0}^{1} G(\sigma(s), s) q_{i}(s) \triangle s+A\left(q_{i}(\cdot)\right)(b+a) B\left(q_{i}(\cdot)\right)(d+c)\right)\left(f_{i \infty}+\epsilon\right)\right]^{-1}\right\} .
$$

Let $T$ be the cone preserving, completely continuous operator that was defined by (3.1). From the definition of $f_{i 0}, 1 \leq i \leq n$, there exists $\bar{H}_{3}>0$ such that, for each $1 \leq i \leq n$,

$$
f_{i}(x) \geq\left(f_{i 0}-\epsilon\right) x, \quad 0<x \leq \bar{H}_{3} .
$$

Also, from the definition of $f_{i 0}$, it follows that $f_{i 0}(0)=0,1 \leq i \leq n$, and so there exist $0<$ $K_{n}<K_{n-1}<\cdots<K_{2}<\bar{H}_{3}$ such that

$$
\lambda_{i} f_{i}(t) \leq \frac{K_{i-1}}{\int_{0}^{1} G(\sigma(s), s) q_{i}(s) \triangle s}, \quad t \in\left[0, K_{i}\right]_{\mathbb{T}}, 3 \leq i \leq n
$$

and

$$
\lambda_{2} f_{2}(t) \leq \frac{\bar{H}_{3}}{\int_{0}^{1} G(\sigma(s), s) q_{2}(s) \triangle s}, \quad t \in\left[0, K_{2}\right]_{\mathbb{T}} .
$$

Choose $u_{1} \in \mathcal{P}$ with $\left\|u_{1}\right\|=K_{n}$. Then we have

$$
\begin{aligned}
& \lambda_{n} \int_{0}^{1} G\left(s_{n-1}, s_{n}\right) q_{n}\left(s_{n}\right) f_{n}\left(u_{1}\left(s_{n}\right)\right) \triangle s_{n} \\
& \leq \lambda_{n} \int_{0}^{1} G\left(\sigma\left(s_{n}\right), s_{n}\right) q_{n}\left(s_{n}\right) f_{n}\left(u_{1}\left(s_{n}\right)\right) \triangle s_{n} \\
& \leq \frac{\int_{0}^{1} G\left(\sigma\left(s_{n}\right), s_{n}\right) q_{n}\left(s_{n}\right) K_{n-1} \triangle s_{n}}{\int_{0}^{1} G\left(\sigma\left(s_{n}\right), s_{n}\right) q_{n}\left(s_{n}\right) \triangle s_{n}} \\
& =K_{n-1} .
\end{aligned}
$$

Continuing with this bootstrapping argument, we get

$$
\begin{aligned}
& \lambda_{2} \int_{0}^{1} G\left(s_{1}, s_{2}\right) q_{2}\left(s_{2}\right) f_{2}\left(\lambda_{3} \int_{0}^{1} G\left(s_{2}, s_{3}\right) q_{3}\left(s_{3}\right) \cdots f_{n}\left(u_{1}\left(s_{n}\right)\right) \triangle s_{n} \cdots \Delta s_{3}\right) \triangle s_{2} \\
& \quad \leq \bar{H}_{3} .
\end{aligned}
$$


Then

$$
\begin{aligned}
T u_{1}(t) & \geq \lambda_{1} \int_{0}^{1} G\left(t, s_{1}\right) q_{1}\left(s_{1}\right) f_{1}\left(\lambda_{2} \int_{0}^{1} G\left(s_{1}, s_{2}\right) q_{2}\left(s_{2}\right) \cdots f_{n}\left(u_{1}\left(s_{n}\right)\right) \triangle s_{n} \cdots \Delta s_{2}\right) \triangle s_{1} \\
& \geq \gamma^{2} \lambda_{1} \int_{\mu}^{1-\mu} G\left(\sigma\left(s_{1}\right), s_{1}\right) q_{1}\left(s_{1}\right)\left(f_{10}-\epsilon\right)\left\|u_{1}\right\| \Delta s_{1} \\
& \geq\left\|u_{1}\right\| .
\end{aligned}
$$

So, $\left\|T u_{1}\right\| \geq\left\|u_{1}\right\|$. If we put

$$
\Omega_{3}=\left\{u \in \mathbb{B} \mid\|u\|<K_{n}\right\},
$$

then

$$
\left\|T u_{1}\right\| \geq\left\|u_{1}\right\| \quad \text { for } u_{1} \in \mathcal{P} \cap \partial \Omega_{3} .
$$

Since each $f_{i \infty}$ is assumed to be a positive real number, it follows that $f_{i}, 1 \leq i \leq n$, is unbounded at $\infty$.

For each $1 \leq i \leq n$, set

$$
f_{i}^{*}(x)=\sup _{0 \leq s \leq x} f_{i}(s)
$$

Then, for each $1 \leq i \leq n, f_{i}^{*}$ is a nondecreasing real-valued function, $f_{i} \leq f_{i}^{*}$, and

$$
\lim _{x \rightarrow \infty} \frac{f_{i}^{*}(x)}{x}=f_{i \infty}
$$

Next, by definition of $f_{i \infty}, 1 \leq i \leq n$, there exists $\bar{H}_{4}$ such that, for each $1 \leq i \leq n$,

$$
f_{i}^{*}(x) \leq\left(f_{i \infty}+\epsilon\right) x, \quad x \geq \bar{H}_{4} .
$$

It follows that there exists $H_{4}>\max \left\{2 \bar{H}_{3}, \bar{H}_{4}\right\}$ such that, for each $1 \leq i \leq n$,

$$
f_{i}^{*}(x) \leq f_{i}^{*}\left(H_{4}\right), \quad 0<x \leq H_{4} .
$$

Choose $u_{1} \in \mathcal{P}$ with $\left\|u_{1}\right\|=H_{4}$. Then, using the bootstrapping argument, we have

$$
\begin{aligned}
& \lambda_{1} \int_{0}^{1} G\left(t, s_{1}\right) q_{1}\left(s_{1}\right) f_{1}\left(\lambda_{2} \cdots\right) \triangle s_{1} \\
& \leq \lambda_{1} \int_{0}^{1} G\left(t, s_{1}\right) q_{1}\left(s_{1}\right) f_{1}^{*}\left(\lambda_{2} \cdots\right) \triangle s_{1} \\
& \quad \leq \lambda_{1} \int_{0}^{1} G\left(\sigma\left(s_{1}\right), s_{1}\right) q_{1}\left(s_{1}\right) f_{1}^{*}\left(H_{4}\right) \triangle s_{1} \\
& \quad \leq \lambda_{1} \int_{0}^{1} G\left(\sigma\left(s_{1}\right), s_{1}\right) q_{1}\left(s_{1}\right) \triangle s_{1}\left(f_{1 \infty}+\epsilon\right) H_{4} .
\end{aligned}
$$


So we have

$$
\begin{aligned}
T u_{1}(t) \leq & \lambda_{1}\left(\int_{0}^{1} G\left(\sigma\left(s_{1}\right), s_{1}\right) q_{1}\left(s_{1}\right) \triangle s_{1}\left(f_{1 \infty}+\epsilon\right) H_{4}\right. \\
& \left.+A\left(q_{1}(\cdot)\right)\left\|f_{1}\left(u_{2}\right)\right\|(b+a)+B\left(q_{1}(\cdot)\right)\left\|f_{1}\left(u_{2}\right)\right\|(d+c)\right) \\
\leq & \lambda_{1}\left(\int_{0}^{1} G\left(\sigma\left(s_{1}\right), s_{1}\right) q_{1}\left(s_{1}\right) \triangle s_{1}\left(f_{1 \infty}+\epsilon\right) H_{4}\right. \\
& \left.+A\left(q_{1}(\cdot)\right)\left\|f_{1}^{*}\left(u_{2}\right)\right\|(b+a)+B\left(q_{1}(\cdot)\right)\left\|f_{1}^{*}\left(u_{2}\right)\right\|(d+c)\right) \\
\leq & \lambda_{1}\left(\int_{0}^{1} G\left(\sigma\left(s_{1}\right), s_{1}\right) q_{1}\left(s_{1}\right) \triangle s_{1}+A\left(q_{1}(\cdot)\right)(b+a)+B\left(q_{1}(\cdot)\right)(d+c)\right) \\
& \times\left(f_{1 \infty}+\epsilon\right) H_{4} \\
\leq & H_{4}=\left\|u_{1}\right\| .
\end{aligned}
$$

Hence, $\left\|T u_{1}\right\| \leq\left\|u_{1}\right\|$. So, if we set

$$
\Omega_{4}=\left\{u \in \mathbb{B} \mid\|u\|<H_{4}\right\},
$$

then

$$
\left\|T u_{1}\right\| \leq\left\|u_{1}\right\| \quad \text { for } u_{1} \in \mathcal{P} \cap \partial \Omega_{4} .
$$

Applying Theorem 2.1 to (3.7) and (3.8), we see that $T$ has a fixed point $u_{1} \in \mathcal{P} \cap\left(\bar{\Omega}_{4} \backslash \Omega_{3}\right)$, which in turn with $u_{n+1}=u_{1}$, we obtain an $n$-tuple $\left(u_{1}, u_{2}, \ldots, u_{n}\right)$ satisfying (1.1)-(1.2) for the chosen values of $\lambda_{i}, 1 \leq i \leq n$. The proof is completed.

\section{An example}

Example 4.1 In BVP (1.1)-(1.2), suppose that $\mathbb{T}=[0,1], n=m=3, q_{1}(t)=q_{2}(t)=q_{3}(t)=1$, $a=c=2, b=d=1, \xi_{1}=\frac{1}{2}, \mu=\frac{1}{4}, \alpha_{1}=\frac{1}{2}$ and $\beta_{1}=3$ i.e.,

$$
\left\{\begin{array}{l}
u_{i}^{\prime \prime}(t)+\lambda_{i} f_{i}\left(u_{i+1}(t)\right)=0, \quad t \in[0,1], 1 \leq i \leq 3 \\
u_{4}(t)=u_{1}(t), \quad t \in[0,1]_{\mathbb{T}}
\end{array}\right.
$$

satisfying the following boundary conditions:

$$
\left\{\begin{array}{l}
2 u_{i}(0)-u_{i}^{\prime}(0)=\frac{1}{2} u_{i}\left(\frac{1}{2}\right), \\
2 u_{i}(1)+u_{i}^{\prime}(1)=3 u_{i}\left(\frac{1}{2}\right), \quad 1 \leq i \leq 3
\end{array}\right.
$$

where

$$
\begin{aligned}
& f_{1}\left(u_{2}\right)=u_{2}\left(1,000-999 e^{-u_{2}}\right)\left(520-512 e^{-2 u_{2}}\right), \\
& f_{2}\left(u_{3}\right)=u_{3}\left(700-698 e^{-2 u_{3}}\right)\left(1,500-1,498 e^{-3 u_{2}}\right), \\
& f_{3}\left(u_{1}\right)=u_{1}\left(800-796 e^{-u_{1}}\right)\left(400-396 e^{-5 u_{1}}\right) .
\end{aligned}
$$


It is easy to see that (C1)-(C7) are satisfied. By simple calculation, we get $\rho=8, \theta(t)=1+2 t$, $\varphi(t)=3-2 t, \Delta=-8, \gamma=\frac{1}{2}, A(1)=9, B(1)=\frac{3}{2}$ and

$$
G(t, s)=\frac{1}{8} \begin{cases}(1+2 s)(3-2 t), & s \leq t, \\ (1+2 t)(3-2 s), & t \leq s .\end{cases}
$$

We obtain

$$
\begin{aligned}
& f_{10}=8, \quad f_{20}=4, \quad f_{30}=16, \\
& f_{1 \infty}=520,000, \quad f_{2 \infty}=1,050,000, \quad f_{3 \infty}=320,000, \\
& M_{1}=\max \{0.0000039279869,0.00000194528875,0.000006382978723\}
\end{aligned}
$$

and

$$
M_{2}=\min \{0.00355450236,0.007109004739,0.001777251184\} .
$$

Applying Theorem 3.1, we get the optimal eigenvalue interval $0.000006382978723<\lambda_{i}<$ $0.001777251184, i=1,2,3$, for which the boundary value problem (4.1)-(4.2) has a positive solution.

\section{Competing interests}

The authors declare that they have no competing interests.

\section{Authors' contributions}

All authors contributed equally to the manuscript and typed, read and approved the final manuscript.

\section{Author details}

'Department of Mathematics, Ege University, Bornova, Izmir, 35100, Turkey. ${ }^{2}$ Department of Mathematics, Gazi University, Teknikokullar, Ankara, 06500, Turkey.

\section{Acknowledgements}

The authors would like to thank the referees for their valuable suggestions and comments.

Received: 3 October 2013 Accepted: 3 March 2014 Published: 21 Mar 2014

\section{References}

1. Hilger, S: Ein maßkettenkalkül mit anwendug auf zentrumsmanningfaltigkeite. PhD thesis, Universität Würzburg (1988)

2. Agarwal, RP, Bohner, M: Basic calculus on time scales and some of its applications. Results Math. 35, 3-22 (1999)

3. Anderson, DR, Karaca, IY: Higher-order three-point boundary value problem on time scales. Comput. Math. Appl. 56, 2429-2443 (2008)

4. Bohner, M, Peterson, A: Dynamic Equations on Time Scales: An Introduction with Applications. Birkhäuser, Boston (2001)

5. Bohner, M, Peterson, A: Advances in Dynamic Equations on Time Scales. Birkhäuser, Boston (2003)

6. Bohner, M, Luo, H: Singular second-order multipoint dynamic boundary value problems with mixed derivatives. Adv. Differ. Equ. 2006, Article ID 54989 (2006)

7. Tokmak, F, Karaca, IY: Existence of symmetric positive solutions for a multipoint boundary value problem with sign-changing nonlinearity on time scales. Bound. Value Probl. 2013, 52 (2013)

8. Agarwal, RP, Bohner, M, Wong, P: Strum-Liouville eigenvalue problems on time scale. Appl. Math. Comput. 99, 153-166 (1999)

9. Anderson, DR: Eigenvalue intervals for even order Strum-Liouville dynamic equations. Commun. Appl. Nonlinear Anal. 12, 1-13 (2005)

10. Benchohra, M, Henderson, J, Ntouyas, SK: Eigenvalue problems for systems of nonlinear boundary value problems on time scales. Adv. Differ. Equ. 2007, Article ID 31640 (2007)

11. Karaca, IY: Multiple positive solutions for dynamic m-point boundary value problems. Dyn. Syst. Appl. 17, 25-42 (2008)

12. Karaca, IY: Existence and nonexistence of positive solutions to a right-focal boundary value problem on time scales. Adv. Differ. Equ. 2006, Article ID 43039 (2006) 
13. Benchohra, M, Berhoun, F, Hamani, S, Henderson, J, Ntouyas, SK, Ouahab, A, Purnaras, IK: Eigenvalues for iterative systems of nonlinear boundary value problems on time scales. Nonlinear Dyn. Syst. Theory 9, 11-22 (2009)

14. Prasad, KR, Sreedhar, N, Narasimhulu, Y: Eigenvalue intervals for iterative systems of nonlinear $m$-point boundary value problems on time scales. Differ. Equ. Dyn. Syst. (2013). doi:10.1007/s12591-013-0183-5

15. Ma, R, Thompson, B: Positive solutions for nonlinear m-point eigenvalue problems. J. Math. Anal. Appl. 297, 24-37 (2004)

16. Guo, D, Lakshmikantham, V: Nonlinear Problems in Abstract Cones. Academic Press, Orlando (1988)

10.1186/1687-2770-2014-63

Cite this article as: Karaca and Tokmak: Eigenvalues for iterative systems of nonlinear $m$-point boundary value problems on time scales. Boundary Value Problems 2014, 2014:63

Submit your manuscript to a SpringerOpen ${ }^{\circ}$ journal and benefit from:

- Convenient online submission

- Rigorous peer review

- Immediate publication on acceptance

- Open access: articles freely available online

- High visibility within the field

- Retaining the copyright to your article

Submit your next manuscript at $>$ springeropen.com 